\title{
THE EFFECT OF SOME REDUCED NITRITE CURING MIXTURES ON SENSORY, PHYSICOCHEMICAL AND MICROBIAL PARAMETERS OF PASTIRMA DURING RIPENING AND STORAGE
}

Mostafa,G.A. a; Amal A. Gaballa a ; R. A. Taha ${ }^{\text {a }}$; S. M. Mokhtar a; B. Nowak ${ }^{b}$ and Theda Von Mueffling ${ }^{b}$

a Dept. of Food Technology, Faculty of Agriculture, Suez Canal University, Ismailia, Egypt

b Institute of Food Quality and Food Safety, University of Veterinary Medicine, Hannover, Germany

\section{ABSTRACT}

For decreasing the use of nitrite in pastirma processing, five nitrite-reduced meat curing mixtures were used as replacements for $32 \%, 64 \%$ and $100 \%$ nitrite. The quantitative characteristics of the samples such as Physicochemical, microbial and sensory attributes were compared with the control (100\% nitrite) during ripening and storage. The statistical comparison showed that curing mixtures which included sodium nitrite $80 \mathrm{mg} / \mathrm{kg}$, chitosan $10 \mathrm{~g} / \mathrm{kg}$ and rosemary $2 \mathrm{~g} / \mathrm{kg}$ (sample B), sodium nitrite $80 \mathrm{mg} / \mathrm{kg}$, betanin $3.6 \mathrm{mg} / \mathrm{kg}$, chitosan $10 \mathrm{~g} / \mathrm{kg}$ and rosemary $2 \mathrm{~g} / \mathrm{kg}$ (sample D) and sodium nitrite $40 \mathrm{mg} / \mathrm{kg}$, betanin $14.4 \mathrm{mg} / \mathrm{kg}$, chitosan $10 \mathrm{~g} / \mathrm{kg}$ and rosemary 2 $\mathrm{g} / \mathrm{kg}$ (sample E) have the color, overall acceptability, oxidative and microbial stabilities which are imparted by nitrite.

Keywords: Curing mixtures, Nitrite, Betanin, Chitosan, Rosemary

\section{INTRODUCTION}

Sodium or potassium nitrite is widely used as curing agent in sausage and other cured meat products because it inhibits outgrowth and neurotoxin formation by Clostridium botulinum, hampers spoilage and the development of food poisoning anaerobic microorganisms, delays the development of oxidative rancidity, develops the characteristic flavor of cured meats and reacts with myoglobin and stabilizes the red meat color. However, nitrite can react with secondary or tertiary amines in meat to form carcinogenic, teratogenic and mutagenic $\mathrm{N}$-nitroso compounds (Honikel, 2008). Despite all of its desired properties, nitrite is also responsible for the formation of carcinogenic $\mathrm{N}$-nitrosamines in some cured meat products under certain processing conditions (Marco et al., 2006). Schweinsberg and Bürkle (1985) reported that nitrite enhances the carcinogenic action of N-nitroso-Nmethylbenzylamine in the production of esophageal tumors.

To overcome these potentially serious problems, several approaches have been considered by researchers. Since the rate of nitrosamine production depends on the square of the concentration of the residual nitrite in meats, a reduction in the level of nitrite addition to meats has proved to be an effective measure in reducing the risk of nitrosamine formation (Shahidi and Pegg, 1992). Therefore, it is desirable to find suitable alternatives for nitrite in the preparation of cured meat products.

Since it is unlikely that a single compound will be found that can perform all of the functions of nitrite, efforts in the past have been 
concentrated to develop nitrite-free curing mixtures for duplicating the cumulative action of nitrite.

The aim of the present research was to develop a multi-component curing system in which individual constituents are used to produce the color, and flavor imparted by nitrite and to reproduce its antioxidant and antimicrobial effects.

\section{MATERIALS AND METHODS}

Materials

Betanin was obtained from Sensient Food Colors (Geesthacht, Germany). Chitosan $75-85 \%$ deacetylation was obtained from Sigma-Aldrich $\mathrm{GmbH}$ (Steinheim, Germany) and Rosemary was purchased from local market (Hannover, Germany). All media used in microbiological analysis were obtained from Merck (Darmstadt, Germany).

Preparation of pastirma

Pastirma was prepared according to the method described by Shehata (1989). During preparation of pastirma the Egyptian standard (1991) was considered. For all treatments $M$. semitendinosus from cows of about the same age (3-4 years old) were used. After that $M$. semitendinosus was trimmed from external fat and connective tissues. Then the muscle $(2-3 \mathrm{~kg})$ was cut into two pieces of approximately 1 to $1.5 \mathrm{~kg}$. The pieces for dry curing received 8 to 10 incisions of $2-3 \mathrm{~cm}$ length and about $2 \mathrm{~cm}$ depth laterally to fill it with curing salt. Sex different batches with different curing mixtures were produced as a follow: $A=$ control (sodium nitrite $125 \mathrm{mg} / \mathrm{kg}$ ), $\mathrm{B}=$ sodium nitrite $80 \mathrm{mg} / \mathrm{kg}$ + chitosan $10 \mathrm{~g} / \mathrm{kg}$ + rosemary $2 \mathrm{~g} / \mathrm{kg}, \mathrm{C}=$ sodium nitrite $40 \mathrm{mg} / \mathrm{kg}+$ chitosan $10 \mathrm{~g} / \mathrm{kg}$ + rosemary $2 \mathrm{~g} / \mathrm{kg}$, D=sodium nitrite 80 $\mathrm{mg} / \mathrm{kg}$ + betanin $3.6 \mathrm{mg} / \mathrm{kg}$ + chitosan $10 \mathrm{~g} / \mathrm{kg}$ + rosemary $2 \mathrm{~g} / \mathrm{kg}$, E=sodium nitrite $40 \mathrm{mg} / \mathrm{kg}$ + betanin $14.4 \mathrm{mg} / \mathrm{kg}$ + chitosan $10 \mathrm{~g} / \mathrm{kg}$ + rosemary $2 \mathrm{~g} / \mathrm{kg}$, and $F=$ =betanin $21.6 \mathrm{mg} / \mathrm{kg}$ + chitosan $10 \mathrm{~g} / \mathrm{kg}$ + rosemary $2 \mathrm{~g} / \mathrm{kg}$. Then all prepared samples were coated (3-4 mm thickness) with the paste mixture (40 $\mathrm{g}$ finely ground fenugreek, $20 \mathrm{~g}$ finely ground garlic, $30 \mathrm{~g}$ paprika, $10 \mathrm{~g}$ sodium chloride, $20 \mathrm{~g}$ wheat flour and $80 \mathrm{ml}$ water). Then the samples were transferred to a chamber (Wilhelm Fessmann GmbH U. Co., Winnenden, Germany), at $60-85 \pm 1 \% \mathrm{RH}$ and at $15-20 \pm 0.5 \stackrel{\circ}{\circ}$ during 17 days according to the drying program described by Aksu et al. (2005), then stored at room temperature $\left(10 \pm 1^{\circ} \mathrm{C}\right)$ for 90 days.

\section{Sampling}

Proximate analyses (moisture, protein, fat and ash) were determined only in the raw meat used for the preparation of pastirma. For tested parameters (moisture, $\mathrm{pH}, \mathrm{TBA}$, color, and nitrite), determinations were performed on $0,1,8$ and 17 days of ripening and 30, 60 and 90 days of storage, microbial examinations were performed on $0,1,4,8$ and 17 days of ripening and 30,60 and 90 days of storage and sensory evaluation was performed directly after ripening and after 30,60 and 90 days of storage. All analyses were carried out in duplicate. 


\section{Physicochemical analysis}

Moisture, crude protein and ash contents were determined according to AOAC (1990). The fat content was determined according to soxhelt method as described by Amtliche Sammlung von Untersuchungsverfahren nach § 64 Lebensmittel-und Futtermittelgesetzbuch (LFGB) (1980). pH value was determined according to the method of Bozkurt (2006). TBA value was determined according to the method described by Shahidi et al. (1987).The color of the sausage samples was measured using Minolta Spectrophotomater CM-2002 (Minolta Camera Co. Ltd., Osaka, Japan), the measurements were repeated on five randomly selected locations on each sample. Residual nitrite was determined according to the method of Amtliche Sammlung von Untersuchungsverfahren nach § 64 LFGB (1990).

\section{Microbiological analysis}

Ten grams of the sample was aseptically weighed into a sterilized plastic bag containing $90 \mathrm{ml}$ diluent $(8.5 \mathrm{~g}$ sodium chloride and $1 \mathrm{~g}$ casein peptone diluted to $1 \mathrm{~L}$ distilled water). Each sample was homogenized with a stomacher 400 lab blender (Fa. Seward Medical, London, UK) for 2 min and the suspension was used as $10^{-1}$ dilution. Serial decimal dilutions were made of sample solution $\left(10^{-2}\right.$ to $\left.10^{-7}\right)$. Total plate count was counted on standard nutrient agar, incubated at $37^{\circ} \mathrm{C}$ for 2 days. Dilutions were spread-plated in duplicate on crystal-violet neutral-red bile dextrose agar (VRBD) for Enterobacteriaceae, Baird Parker agar (BP) Micrococcaceae, Escherichia coli direct agar (ECD) for E. coli and yeast extract glucose chloramphenicol agar (YGC) for yeast and mould. Plates were incubated at $30{ }^{\circ} \mathrm{C}$ for 2 days for Enterobacteriaceae, $37^{\circ} \mathrm{C}$ for 2 days for Micrococcaceae, $37^{\circ} \mathrm{C}$ for 2 days for $E$. coli and $25^{\circ} \mathrm{C}$ for 4 days for yeast and mould.

\section{Sensory evaluation}

The organoleptic quality attributes (appearance, color, texture, odor and taste) of the control and treated samples were evaluated initially and periodically during storage. Sensory evaluation was performed with 10 trained panel members, Institute of Food Quality and Food Safety, University of Veterinary Medicine, Hannover, Germany. The panelists were asked to evaluate each attribute on a 5-point scale: 1, very poor; 2, poor; 3, acceptable; 4, good; 5 , very good according to the method described by Byun et al. (2001).

\section{Statistical analysis}

Significant differences between the mean values of estimated testes were measured according to Strotmann et al. (2008).

\section{RESULTS AND DISCUSSION}

\section{Physicochemical analysis}

Mean percent contents of moisture, protein, fat and ash of the raw meat used for the preparation of pastirma were $73.5 \%, 23.0 \%, 2.25 \%$ and $1.05 \%$, respectively. These results are close to those reported by Aksu et al. (2005) for Pastirma.Changes in the moisture content during ripening and storage are shown in Fig. (1a). The moisture content of pastirma samples 
gradually decreased during ripening and storage periods as a result of drying and water evaporation. On the day of stuffing mean values for moisture were above $69 \%$ for all batters (from $59.38 \%$ to $70.7 \%$ ), whereas at the end of ripening (day 17) it was above $47 \%$ (from $47.9 \%$ t0 $49.0 \%$ ). At the end of storage (day 90), the mean values were over $40 \%(40.9 \%$ to $41.6 \%)$.Changes of $\mathrm{pH}$ during the ripening and storage periods of pastirma samples are given in Fig. (1b). $\mathrm{pH}$ values of pastirma decreased during the first 8 days of ripening from 5.94 to 5.57 . During this period, lactic acid bacteria and other acid-producing bacteria produce lactic acid and other organic acids (Komprda et al., 2001). After that time during further ripening and storage, an increase in $\mathrm{pH}$ value was observed and this may be due to decomposition of acids and production of basic nitrogenous compounds (Bozkurt and Erkmen, 2007). It was found that additives affected $(P<0.05)$ $\mathrm{pH}$ values of pastirma samples. Increasing nitrite concentration decreased ( $\mathrm{P}$ $<0.05) \mathrm{pH}$ values (Bozkurt and Erkmen, 2007).

Changes of TBA values of pastirma samples during the ripening and storage are given in Fig (1c). It was found that TBA values were did not affected $(P<0.05)$ by nitrite alternatives. TBA values increased gradually $(P$ $<0.05$ ) from 0.15 to $0.52 \mathrm{mg}$ malonaldehyde/ $\mathrm{kg}$ during the ripening period. After that, TBA values increased during the storage period up to $0.71 \mathrm{mg}$ malonaldehyde/kg. In agreement with the obtained results, the positive effects of the individual use of chitosan and rosemary or the combined use as regards preventation of lipid oxidation are well documented. Georgantelis et al. (2007) found that the best antioxidative effect in sausage and beef burger was obtained by the combination of rosemary extract and chitosan. Also, Darmadji and Izumimuto (1994) reported that the TBA value of beef containing $1 \%$ chitosan was at the same level after 10 days of storage at 4 ${ }^{\circ} \mathrm{C}$ as it was on day 0 , whereas the respective value of the control samples increased sharply.Changes in the residual nitrite level of pastirma samples are shown in Fig. (1d). The residual nitrite levels decreased rapidly during ripening and decreased slowly during storage. At any sampling time during ripening and storage the control sample (A) showed significantly higher $(p<0.05)$ levels of residual nitrite than those observed in the other samples (B, C, D, E and F). Alley et al.(1992) observed that in the first stages of fermentation, more than $50 \%$ of nitrites that disappeared were transformed into nitrates. The rapid decrease in nitrite level observed in dry sausages is well documented (Samelis et al., 1998). The nitrite content decreased very quickly which is most likely due to the high reactivity of nitrite (Marco et al., 2006). Aksu and Kaya (2001) reported that the amount of residual nitrite of pastirma marketed in Erzurum, Turkey, was $0.93-11.6 \mathrm{mg} / \mathrm{kg}$. Tyrpenou et al. (2000) found that the amount of residual nitrite in Greek pastirma ranged from 0.85 to $190 \mathrm{mg} / \mathrm{kg}$ as sodium nitrite.

Changes in $L, a$ and $b$ values of pastirma samples during the ripening and storage periods are given in Table (1). Significant differences $(p<0.05)$ were found between betanin containing samples $(D, E$ and $F)$ and free betanin samples (A, B and $C$ ). The lightness ( $L$ value) of all pastirma samples was slowly decreased during ripening and storage. 
Fig. (1). Effect of different curing mixtures on physicochemical properties of pastirma during ripening and storage.

A: control sodium nitrite $125 \mathrm{mg} / \mathrm{kg}$, B: sodium nitrite $80 \mathrm{mg} / \mathrm{kg}+$ chitosan10 g/kg + rosemary $2 \mathrm{~g} / \mathrm{kg}$, C: sodium nitrite $40 \mathrm{mg} / \mathrm{kg} \mathrm{+}$ chitosan $10 \mathrm{~g} / \mathrm{kg}$ + rosemary $2 \mathrm{~g} / \mathrm{kg}$, D: sodium nitrite $80 \mathrm{mg} / \mathrm{kg}$ + betanin $3.6 \mathrm{mg} / \mathrm{kg}$ + chitosan $10 \mathrm{~g} / \mathrm{kg}$ + rosemary $2 \mathrm{~g} / \mathrm{kg}$, E: sodium nitrite $40 \mathrm{mg} / \mathrm{kg}$ + betanin $14.4 \mathrm{mg} / \mathrm{kg}$ + chitosan $10 \mathrm{~g} / \mathrm{kg}$ + rosemary 2 $\mathrm{g} / \mathrm{kg}$, F: betanin $21.6 \mathrm{mg} / \mathrm{kg}+$ chitosan $10 \mathrm{~g} / \mathrm{kg}+$ rosemary $2 \mathrm{~g} / \mathrm{kg}$. 
Mostafa, G. A. et al.

Decrease in $L$ values represented formation of dark color in the sucuk due to the browning reactions (Bozkurt, 2006). These results are in accordance with those reported by Chouliara et al. (2006). Aksu et al. (2005) who reported that $L$ values decreased during storage from 47.38 to 41.42 during the storage period. Significant differences $(p<0.05)$ for a values were observed between the investigated samples, on the day of stuffing sample $(F)$ produced the highest $a$ value among the samples, while samples ( $B$ and $C$ ) revealed the lowest values. Bloukas et al. (1999) reported that the redness of frankfurters was steadily increased with the betanin level. Similar results were confirmed by Stuempel (1997) for betanin in frankfurter-type sausages. An increasing trend was observed as regards to a values for $A, B, C, D$ and $E$ samples, together with a decreasing trend in a values for $F$ sample during ripening. These results are in agreement with those reported by Aksu et al. (2005) who mentioned that a value increased from 24.47 to 36.38 during ripening. During the first days of the ripening period, nitrogenous compounds present in meat combined with myoglobin to produce the desired red pigment (Bozkurt, 2006). a values in all samples decreased as the time of storage increased up to 90 days of storage. These results are in agreement with those observed by several authors (Mitsumoto et al., 2005). The yellowness ( $b$ values) decreased gradually during ripening and storage. This could be due to the browning reactions, because browning reactions yield melanoidins that have a brown color (Bozkurt, 2006). These results are in agreement with those observed by Kayaardi and Gök (2003) and Bozkurt (2006). The $b$ values of Spanish type dry-cured sausages decreased during the fermentation and ripening periods (Pérez-Alvarez et al., 1999). Also, Zarringhalami et al. (2009) reported that the $b$ values of sausage samples decreased during storage as the time of storage increased.

\subsection{Microbiological analysis}

The microbiological analysis was determined during the ripening and storage periods and results are given in Table (2). Counts of TPC and Micrococcaceae increased during the first 8 days of ripening from 6.77 to 8.27 and from 4.21 to $5.15 \mathrm{log} \mathrm{CFU} / \mathrm{g}$, respectively, afterwards decreased during further ripening and storage. At the end of storage, the mean values were 6.10 and $4.20 \log \mathrm{CFU} / \mathrm{g}$, respectively. These results are coincided with those observed by Bozkurt and Erkmen (2007) who found that APC increased from 5.19 to $6.09 \log \mathrm{CFU} / \mathrm{g}$ during the first 10 days of the ripening period, due to higher $\mathrm{RH}(85-95 \%)$ and temperature $\left(22-25^{\circ} \mathrm{C}\right)$.

After that, it decreased significantly $(P>0.05)$, during storage, due to adjustment of $\mathrm{RH}$ to $50 \% \mathrm{RH}$. Enterobacteriaceae counts decreased during ripening by more than one logarithmic unit, ending up at less than 3 log $\mathrm{CFU} / \mathrm{g}$ in all batches. Also, Enterobacteriaceae counts of all tested pastirma samples decreased as the storage time progressed up to 30 days. While, no Enterobacteriaceae colonies were observed at 60 and 90 days of storage. This behavior is similar to that found by Bover-Cid et al. (1999).This is a typical decrease due to the environmental conditions which make Gramnegative bacteria growth difficult (Hernández-Jover et al., 1997). 
J. Agric. Sci. Mansoura Univ., 34 (7): 7715 -7726, 2009 1 
Table (2). Effect of different curing mixtures on microbiological profiles of pastirma during ripening and storage.

\begin{tabular}{|c|c|c|c|c|c|c|}
\hline Time (days) & Treatment & $\begin{array}{c}\text { Total plate } \\
\text { count }\end{array}$ & Enterobacteriaceae & $\begin{array}{l}\text { Lactic acid } \\
\text { bacteria }\end{array}$ & $\begin{array}{l}\text { Yeast and } \\
\text { mould }\end{array}$ & E. coli \\
\hline $\begin{array}{c}\text { Ripening } \\
0\end{array}$ & $\begin{array}{l}\text { A } \\
\text { B } \\
\text { C } \\
\text { D } \\
\text { E } \\
\text { F }\end{array}$ & $\begin{array}{l}6.78 \pm 0.085 a \\
6.73 \pm 0.078 a \\
6.79 \pm 0.057 a \\
6.70 \pm 0.042 a \\
6.80 \pm 0.078 a \\
6.82 \pm 0.106 a\end{array}$ & $\begin{array}{l}4.08 \pm 0.057 a \\
4.10 \pm 0.042 a \\
4.10 \pm 0.035 a \\
4.07 \pm 0.078 a \\
4.12 \pm 0.042 a \\
4.14 \pm 0.099 a\end{array}$ & $\begin{array}{l}3.42 \pm 0.042 a \\
3.38 \pm 0.099 a \\
3.45 \pm 0.078 a \\
3.40 \pm 0.049 a \\
3.34 \pm 0.085 a \\
3.31 \pm 0.057 a\end{array}$ & $\begin{array}{l}3.73 \pm 0.106 a \\
3.70 \pm 0.127 a \\
3.75 \pm 0.057 a \\
3.71 \pm 0.078 a \\
3.79 \pm 0.112 a \\
3.79 \pm 0.066 a\end{array}$ & $\begin{array}{l}1.85 \pm 0.211 a \\
1.75 \pm 0.116 a \\
1.82 \pm 0.303 a \\
1.70 \pm 0.087 a \\
1.85 \pm 0.077 a \\
1.90 \pm 0.066 a\end{array}$ \\
\hline 1 & $\begin{array}{l}\text { A } \\
\text { B } \\
\text { C } \\
\text { D } \\
\text { E } \\
\text { F }\end{array}$ & $\begin{array}{l}7.23 \pm 0.211 a \\
7.22 \pm 0.106 a \\
7.18 \pm 0.078 a \\
7.19 \pm 0.085 a \\
7.29 \pm 0.116 a \\
7.37 \pm 0.212 a\end{array}$ & $\begin{array}{l}4.28 \pm 0.217 a \\
4.32 \pm 0.112 a \\
4.30 \pm 0.106 a \\
4.20 \pm 0.092 a \\
4.19 \pm 0.078 a \\
4.30 \pm 0.085 a\end{array}$ & $\begin{array}{l}3.92 \pm 0.092 a \\
3.71 \pm 0.069 a \\
3.88 \pm 0.085 a \\
3.74 \pm 0.049 a \\
3.80 \pm 0.066 a \\
3.95 \pm 0.078 a\end{array}$ & \begin{tabular}{|l|}
$3.94 \pm 0.100 a$ \\
$3.88 \pm 0.120 a$ \\
$3.99 \pm 0.066 a$ \\
$3.96 \pm 0.072 a$ \\
$4.03 \pm 0.215 a$ \\
$4.01 \pm 0.049 a$ \\
\end{tabular} & $\begin{array}{l}1.59 \pm 0.495 a \\
1.58 \pm 0.240 a \\
1.55 \pm 0.099 a \\
1.50 \pm 0.071 a \\
1.55 \pm 0.099 a \\
1.60 \pm 0.212 a\end{array}$ \\
\hline 4 & $\begin{array}{l}\text { A } \\
\text { B } \\
\text { C } \\
\text { D } \\
\text { E } \\
\text { F }\end{array}$ & $\begin{array}{l}7.97 \pm 0.156 a \\
7.89 \pm 0.174 a \\
7.99 \pm 0.099 a \\
7.85 \pm 0.057 a \\
8.01 \pm 0.078 a \\
8.03 \pm 0.066 a\end{array}$ & $\begin{array}{c}4.16 \pm 0.207 a \\
4.06 \pm 0.232 a \\
4.14 \pm 0.057 a \\
4.01 \pm 0.091 a \\
4.09 \pm 0.156 a \\
4.22 \pm 0.066 a\end{array}$ & $\begin{array}{l}5.45 \pm 0.035 a \\
5.26 \pm 0.039 a \\
5.41 \pm 0.074 a \\
5.32 \pm 0.042 a \\
5.38 \pm 0.089 a \\
5.47 \pm 0.127 a\end{array}$ & $\begin{array}{l}5.07 \pm 0.033 a \\
4.85 \pm 0.276 a \\
5.03 \pm 0.042 a \\
4.98 \pm 0.116 a \\
4.99 \pm 0.074 a \\
5.12 \pm 0.202 a\end{array}$ & $\begin{array}{l}1.17 \pm 0.212 a \\
1.12 \pm 0.127 a \\
1.15 \pm 0.106 a \\
1.10 \pm 0.089 a \\
1.17 \pm 0.099 a \\
1.22 \pm 0.127 a\end{array}$ \\
\hline 8 & $\begin{array}{l}\text { A } \\
\text { B } \\
\text { C } \\
\text { D } \\
\text { E } \\
\text { F }\end{array}$ & $\begin{array}{l}8.23 \pm 0.211 a \\
8.08 \pm 0.080 a \\
8.29 \pm 0.137 a \\
8.15 \pm 0.078 a \\
8.37 \pm 0.116 a \\
8.48 \pm 0.232 a\end{array}$ & $\begin{array}{l}3.55 \pm 0.215 a \\
3.48 \pm 0.087 a \\
3.55 \pm 0.091 a \\
3.40 \pm 0.085 a \\
3.45 \pm 0.112 a \\
3.65 \pm 0.156 a\end{array}$ & $\begin{array}{l}6.24 \pm 0.057 a \\
6.12 \pm 0.106 a \\
6.19 \pm 0.057 a \\
6.09 \pm 0.099 a \\
6.17 \pm 0.066 a \\
6.29 \pm 0.212 a\end{array}$ & $\begin{array}{l}4.26 \pm 0.033 a \\
4.14 \pm 0.071 a \\
4.18 \pm 0.049 a \\
4.12 \pm 0.207 a \\
4.22 \pm 0.091 a \\
4.30 \pm 0.349 a\end{array}$ & $\begin{array}{l}1.09 \pm 0.085 a \\
1.00 \pm 0.092 a \\
1.02 \pm 0.112 a \\
1.02 \pm 0.142 a \\
1.05 \pm 0.232 a \\
1.11 \pm 0.149 a\end{array}$ \\
\hline 17 & $\begin{array}{l}\text { A } \\
\text { B } \\
\text { C } \\
\text { D } \\
\text { E } \\
\text { F }\end{array}$ & $\begin{array}{l}8.01 \pm 0.114 a \\
7.98 \pm 0.066 a \\
8.09 \pm 0.078 a \\
8.03 \pm 0.091 a \\
8.11 \pm 0.126 a \\
8.22 \pm 0.111 a\end{array}$ & $\begin{array}{l}2.82 \pm 0.106 a \\
2.72 \pm 0.078 a \\
2.85 \pm 0.042 a \\
2.79 \pm 0.099 a \\
2.80 \pm 0.057 a \\
2.89 \pm 0.091 a\end{array}$ & $\begin{array}{l}5.09 \pm 0.085 a \\
4.88 \pm 0.078 a \\
5.04 \pm 0.057 a \\
5.00 \pm 0.089 a \\
5.02 \pm 0.127 a \\
5.12 \pm 0.349 a\end{array}$ & $\begin{array}{l}3.20 \pm 0.215 a \\
3.01 \pm 0.156 a \\
3.15 \pm 0.085 a \\
3.08 \pm 0.099 a \\
3.10 \pm 0.057 a \\
3.23 \pm 0.174 a\end{array}$ & $\begin{array}{l}\text { ND } \\
\text { ND } \\
\text { ND } \\
\text { ND } \\
\text { ND } \\
\text { ND }\end{array}$ \\
\hline $\begin{array}{r}\text { Storage } \\
0\end{array}$ & $\begin{array}{l}\text { A } \\
\text { B } \\
\text { C } \\
\text { D } \\
\text { E } \\
\text { F }\end{array}$ & $\begin{array}{l}8.01 \pm 0.114 a \\
7.98 \pm 0.066 a \\
8.09 \pm 0.078 a \\
8.03 \pm 0.091 a \\
8.11 \pm 0.126 a \\
8.22 \pm 0.111 a\end{array}$ & $\begin{array}{l}2.82 \pm 0.106 a \\
2.72 \pm 0.078 a \\
2.85 \pm 0.042 a \\
2.79 \pm 0.099 a \\
2.80 \pm 0.057 a \\
2.89 \pm 0.091 a\end{array}$ & $\begin{array}{l}5.09 \pm 0.085 a \\
4.88 \pm 0.078 a \\
5.04 \pm 0.057 a \\
5.00 \pm 0.089 a \\
5.02 \pm 0.127 a \\
5.12 \pm 0.349 a\end{array}$ & $\begin{array}{l}3.20 \pm 0.215 a \\
3.01 \pm 0.156 a \\
3.15 \pm 0.085 a \\
3.08 \pm 0.099 a \\
3.10 \pm 0.057 a \\
3.23 \pm 0.174 a\end{array}$ & $\begin{array}{l}\text { ND } \\
\text { ND } \\
\text { ND } \\
\text { ND } \\
\text { ND } \\
\text { ND }\end{array}$ \\
\hline 30 & $\begin{array}{l}\text { A } \\
\text { B } \\
\text { C } \\
\text { D } \\
\text { E } \\
\text { F }\end{array}$ & $\begin{array}{l}7.68 \pm 0.092 a \\
7.77 \pm 0.111 a \\
7.71 \pm 0.087 a \\
7.60 \pm 0.089 a \\
7.79 \pm 0.091 a \\
7.89 \pm 0.116 a\end{array}$ & $\begin{array}{l}2.35 \pm 0.074 a \\
2.13 \pm 0.240 a \\
2.23 \pm 0.091 a \\
2.21 \pm 0.129 a \\
2.30 \pm 0.137 a \\
2.36 \pm 0.320 a\end{array}$ & $\begin{array}{l}4.34 \pm 0.099 a \\
4.12 \pm 0.085 a \\
4.18 \pm 0.108 a \\
4.20 \pm 0.099 a \\
4.29 \pm 0.106 a \\
4.36 \pm 0.137 a\end{array}$ & $\begin{array}{l}2.29 \pm 0.156 a \\
2.14 \pm 0.087 a \\
2.25 \pm 0.114 a \\
2.20 \pm 0.085 a \\
2.28 \pm 0.112 a \\
2.35 \pm 0.273 a\end{array}$ & $\begin{array}{l}\text { ND } \\
\text { ND } \\
\text { ND } \\
\text { ND } \\
\text { ND } \\
\text { ND }\end{array}$ \\
\hline 60 & $\begin{array}{l}\text { A } \\
\text { B } \\
\text { C } \\
\text { D } \\
\text { E } \\
\text { F }\end{array}$ & $\begin{array}{l}6.69 \pm 0.207 a \\
6.66 \pm 0.215 a \\
6.82 \pm 0.108 a \\
6.56 \pm 0.099 a \\
6.72 \pm 0.114 a \\
6.89 \pm 0.127 a\end{array}$ & $\begin{array}{l}\text { ND } \\
\text { ND } \\
\text { ND } \\
\text { ND } \\
\text { ND } \\
\text { ND }\end{array}$ & $\begin{array}{l}3.88 \pm 0.215 a \\
3.62 \pm 0.116 a \\
3.71 \pm 0.156 a \\
3.70 \pm 0.092 a \\
3.75 \pm 0.174 a \\
3.97 \pm 0.230 a\end{array}$ & $\begin{array}{l}2.19 \pm 0.112 a \\
2.01 \pm 0.057 a \\
2.20 \pm 0.099 a \\
2.07 \pm 0.085 a \\
2.15 \pm 0.078 a \\
2.26 \pm 0.089 a\end{array}$ & $\begin{array}{l}\text { ND } \\
\text { ND } \\
\text { ND } \\
\text { ND } \\
\text { ND } \\
\text { ND }\end{array}$ \\
\hline 90 & $\begin{array}{l}\text { A } \\
\text { B } \\
\text { C } \\
\text { D } \\
\text { E } \\
\text { F }\end{array}$ & $\begin{array}{l}6.12 \pm 0.085 a \\
6.09 \pm 0.042 a \\
6.09 \pm 0.066 a \\
6.02 \pm 0.099 a \\
6.10 \pm 0.078 a \\
6.15 \pm 0.057 a\end{array}$ & $\begin{array}{l}\text { ND } \\
\text { ND } \\
\text { ND } \\
\text { ND } \\
\text { ND } \\
\text { ND }\end{array}$ & $\begin{array}{l}3.42 \pm 0.303 a \\
3.28 \pm 0.116 a \\
3.38 \pm 0.350 a \\
3.30 \pm 0.137 a \\
3.35 \pm 0.330 a \\
3.46 \pm 0.120 a\end{array}$ & $\begin{array}{l}2.09 \pm 0.078 a \\
1.73 \pm 0.156 a \\
1.91 \pm 0.127 a \\
1.86 \pm 0.099 a \\
1.97 \pm 0.085 a \\
2.16 \pm 0.112 a\end{array}$ & $\begin{array}{l}\text { ND } \\
\text { ND } \\
\text { ND } \\
\text { ND } \\
\text { ND } \\
\text { ND }\end{array}$ \\
\hline
\end{tabular}

Values with different letters in each column are significantly different $(p<0.05)$ from one to another. (A: control sodium nitrite $125 \mathrm{mg} / \mathrm{kg}$, B: sodium nitrite $80 \mathrm{mg} / \mathrm{kg}$ + betanin 3.6 $\mathrm{mg} / \mathrm{kg}$ + chitosan $10 \mathrm{~g} / \mathrm{kg}$ + rosemary $2 \mathrm{~g} / \mathrm{kg}$, C: sodium nitrite $40 \mathrm{mg} / \mathrm{kg}$ + betanin 14.4 $\mathrm{mg} / \mathrm{kg}$ + chitosan $10 \mathrm{~g} / \mathrm{kg}$ + rosemary $2 \mathrm{~g} / \mathrm{kg}$, D: betanin $21.6 \mathrm{mg} / \mathrm{kg}$ + chitosan $10 \mathrm{~g} / \mathrm{kg}+$ rosemary $2 \mathrm{~g} / \mathrm{kg}$ ). 
Yeast and mould numbers increased from 3.75 to $5.01 \mathrm{log}$ CFU/g up to 4 days of ripening and after that their numbers decreased to 3.13 and 1.95 $\log \mathrm{CFU} / \mathrm{g}$ at the end of ripening and storage, respectively. This trend is similar to that reported by Bozkurt and Erkmen (2007) who found that yeast and mould count increased from 4.54 to $5.09 \mathrm{log}$ CFU/g up to 5 days of ripening and after that their count decreased. E. coli count in all treated samples was gradually decreased during the first 8 days of ripening. Thereafter, no $E$. coli colonies were detected during further ripening and storage.

\section{Sensory evaluation}

Changes in sensory attributes after ripening and during storage of pastirma are given in Table (3).

Table (3). Effect of different curing systems on organoleptic properties of pastirma during storage.

\begin{tabular}{|c|c|c|c|c|c|c|}
\hline \multirow[t]{2}{*}{ Treatments } & Appearance & Color & Texture & Odor & Taste & $\begin{array}{c}\text { Overall } \\
\text { acceptability }\end{array}$ \\
\hline & \multicolumn{6}{|c|}{ Zero time } \\
\hline $\mathbf{A}$ & $4.35 \pm 0.21 a$ & $4.50 \pm 0.20 a$ & $4.28 \pm 0.12 a$ & $4.42 \pm 0.23 a$ & $4.25 \pm 0.21 a$ & $4.36 \pm 0.10 a$ \\
\hline B & $4.10 \pm 0.21 \mathrm{ab}$ & $4.22 \pm 0.28 \mathrm{abc}$ & $4.23 \pm 0.16 a$ & $4.32 \pm 0.26 a$ & $4.10 \pm 0.26 a$ & $4.19 \pm 0.12 \mathrm{ab}$ \\
\hline C & $4.00 \pm 0.14 a b$ & $3.76 \pm 0.09 b c$ & $4.02 \pm 0.26 a$ & $4.05 \pm 0.21 a$ & $4.01 \pm 0.18 a$ & $3.97 \pm 0.07 b c$ \\
\hline D & $4.42 \pm 0.26 a$ & $4.49 \pm 0.21 \mathrm{a}$ & $4.31 \pm 0.27 a$ & $4.49 \pm 0.26 a$ & $4.12 \pm 0.17 a$ & $4.37 \pm 0.14 a$ \\
\hline $\mathbf{E}$ & $4.19 \pm 0.18 a b$ & $4.38 \pm 0.26 a b$ & $4.23 \pm 0.16 a$ & $4.36 \pm 0.20 a$ & $4.13 \pm 0.18 a$ & $4.26 \pm 0.11 a$ \\
\hline $\mathbf{F}$ & $3.70 \pm 0.16 b$ & $3.61 \pm 0.14 c$ & $4.01 \pm 0.30 \mathrm{a}$ & $3.91 \pm 0.26 a$ & $3.96 \pm 0.27 a$ & $3.84 \pm 0.07 \mathrm{c}$ \\
\hline \multicolumn{7}{|c|}{30 days } \\
\hline $\mathbf{A}$ & $4.08 \pm 0.21 \mathrm{ab}$ & $4.18 \pm 0.14 a$ & $4.11 \pm 0.16 a$ & $4.08 \pm 0.20 a$ & $4.27 \pm 0.16 a$ & $4.14 \pm 0.12 a$ \\
\hline B & $3.89 \pm 0.20 a b c$ & $4.02 \pm 0.18 a b$ & $4.03 \pm 0.21 \mathrm{a}$ & $4.07 \pm 0.26 a$ & $4.03 \pm 0.21 \mathrm{ab}$ & $4.01 \pm 0.13 a b$ \\
\hline C & $3.71 \pm 0.07 \mathrm{bc}$ & $3.80 \pm 0.11 b c$ & $3.96 \pm 0.17 a$ & $3.78 \pm 0.23 a b$ & $3.90 \pm 0.14 \mathrm{ab}$ & $3.83 \pm 0.07 \mathrm{bc}$ \\
\hline D & $4.03 \pm 0.10 a$ & $4.20 \pm 0.14 a$ & $4.10 \pm 0.20 a$ & $4.17 \pm 0.21 a$ & $4.19 \pm 0.21 a$ & $4.14 \pm 0.14 a b$ \\
\hline $\mathbf{E}$ & $3.88 \pm 0.14 a b c$ & $4.00 \pm 0.13 a b$ & $3.96 \pm 0.21 \mathrm{a}$ & $3.91 \pm 0.20 a$ & $3.89 \pm 0.16 \mathrm{ab}$ & $3.93 \pm 0.14 \mathrm{abc}$ \\
\hline $\mathbf{F}$ & $3.41 \pm 0.20 \mathrm{~b}$ & $3.38 \pm 0.22 c$ & $3.82 \pm 0.20 a$ & $3.34 \pm 0.04 b$ & $3.70 \pm 0.10 \mathrm{~b}$ & $3.53 \pm 0.14 c$ \\
\hline \multicolumn{7}{|c|}{60 days } \\
\hline $\mathbf{A}$ & $3.83 \pm 0.18 a$ & $3.95 \pm 0.21 a$ & $3.81 \pm 0.16 a$ & $3.71 \pm 0.18 \mathrm{ab}$ & $3.80 \pm 0.21 \mathrm{a}$ & $3.82 \pm 0.14 a$ \\
\hline B & $3.46 \pm 0.21 \mathrm{abc}$ & $3.81 \pm 0.18 a$ & $3.53 \pm 0.18 \mathrm{ab}$ & $3.56 \pm 0.14 a b c$ & $3.72 \pm 0.17 a$ & $3.62 \pm 0.17 a b$ \\
\hline C & $3.26 \pm 0.09 b c$ & $3.30 \pm 0.12 b$ & $3.46 \pm 0.16 \mathrm{ab}$ & $3.27 \pm 0.14 \mathrm{ac}$ & $3.42 \pm 0.21 \mathrm{ab}$ & $3.34 \pm 0.11 \mathrm{bcd}$ \\
\hline D & $3.73 \pm 0.18 a$ & $3.88 \pm 0.17 a$ & $3.60 \pm 0.21 \mathrm{ab}$ & $3.79 \pm 0.11 b$ & $3.83 \pm 0.18 a$ & $3.77 \pm 0.14 a$ \\
\hline $\mathbf{E}$ & $3.62 \pm 0.17 a b$ & $3.83 \pm 0.23 a b$ & $3.40 \pm 0.14 a b$ & $3.62 \pm 0.17 \mathrm{abc}$ & $3.70 \pm 0.14 \mathrm{a}$ & $3.63 \pm 0.18 a c$ \\
\hline $\mathbf{F}$ & $3.01 \pm 0.16 c$ & $2.82 \pm 0.11 \mathrm{c}$ & $3.15 \pm 0.13 b$ & $3.20 \pm 0.12 \mathrm{c}$ & $3.00 \pm 0.21 b$ & $3.04 \pm 0.14 d$ \\
\hline \multicolumn{7}{|c|}{90 days } \\
\hline $\mathbf{A}$ & $3.36 \pm 0.18 a$ & $3.76 \pm 0.17 a$ & $3.06 \pm 0.34 a$ & $3.84 \pm 0.28 a$ & $3.64 \pm 0.16 a$ & $3.53 \pm 0.14 a$ \\
\hline B & $3.12 \pm 0.17 a b$ & $3.32 \pm 0.14 a b$ & $3.07 \pm 0.23 a$ & $3.64 \pm 0.28 \mathrm{ab}$ & $3.59 \pm 0.14 a$ & $3.35 \pm 0.12 a b$ \\
\hline C & $3.01 \pm 0.14 a b$ & $3.02 \pm 0.10 b c$ & $3.09 \pm 0.16 a$ & $3.38 \pm 0.21 \mathrm{ab}$ & $3.25 \pm 0.07 b$ & $3.15 \pm 0.11 \mathrm{bcd}$ \\
\hline D & $3.35 \pm 0.16 a$ & $3.65 \pm 0.14 a$ & $3.18 \pm 0.26 a$ & $3.68 \pm 0.22 a b$ & $3.67 \pm 0.16 a$ & $3.51 \pm 0.14 \mathrm{a}$ \\
\hline $\mathbf{E}$ & $3.17 \pm 0.17 a b$ & $3.41 \pm 0.11 \mathrm{a}$ & $3.23 \pm 0.28 a$ & $3.53 \pm 0.18 a b$ & $3.54 \pm 0.14 \mathrm{ab}$ & $3.38 \pm 0.16 a c$ \\
\hline $\mathbf{F}$ & $2.74 \pm 0.14 b$ & $2.85 \pm 0.10 c$ & $2.96 \pm 0.20 \mathrm{a}$ & $2.98 \pm 0.17 a$ & $2.94 \pm 0.11 \mathrm{c}$ & $2.89 \pm 0.09 d$ \\
\hline
\end{tabular}

Values with different letters in each column are significantly different $(p<0.05)$ from one to another. (A: control sodium nitrite $125 \mathrm{mg} / \mathrm{kg}$, , B: sodium nitrite $80 \mathrm{mg} / \mathrm{kg}+$ chitosan 10 g/kg+rosemary 2000 mg/kg, C: sodium nitrite 40 mg/kg+chitosan 10 g/kg+rosemary 2000 $\mathrm{mg} / \mathrm{kg}$, D: sodium nitrite $80 \mathrm{mg} / \mathrm{kg}+$ betanin $3.6 \mathrm{mg} / \mathrm{kg}+$ chitosan $10 \mathrm{~g} / \mathrm{kg}+$ rosemary 2000 $\mathrm{mg} / \mathrm{kg}$, E: sodium nitrite $40 \mathrm{mg} / \mathrm{kg}$ +betanin $14.4 \mathrm{mg} / \mathrm{kg}+$ chitosan $10 \mathrm{~g} / \mathrm{kg}+$ rosemary 2000 $\mathrm{mg} / \mathrm{kg}$, F: betanin $21.6 \mathrm{mg} / \mathrm{kg}+$ chitosan $10 \mathrm{~g} / \mathrm{kg}+$ rosemary $2000 \mathrm{mg} / \mathrm{kg}$ ).

Sensory scale: 1 , very poor; 2 , poor; 3 , acceptable; 4 , good; 5 , very good.

No significant differences with respect to texture, odor and taste between batches were observed at the end of the ripening process (zero time 
storage), while significant differences $(P<0.05)$ were found in appearance, color and overall acceptability showing a preference for $\mathrm{B}, \mathrm{A}, \mathrm{D}$ and $\mathrm{E}$ samples. These results are in accordance with those observed by Bakr and Mahmoud (1995) who mentioned that sausages prepared with curing mixture including $0.2-0.3 \%$ nitrite, $0.05-0.1 \%$ table beet juice and $0.25 \%$ rosemary extract possessed similar organoleptic qualities as the nitrite cured ones $(0.4$ $\%)$. During storage, it could be noticed that the score of all tested attributes decreased as the storage time progressed up to 90 days. This behavior could be due to lipid, $\mathrm{Mb}$ and nitrosomyoglobin oxidation, as well as microbial activity. Oxidative processes in meat lead to the degradation of lipids and proteins which, in turn, contribute to the deterioration in flavor, texture and color of displayed meat (Decker et al., 1995). At the end of storage time sample (A) recorded the highest score values of appearance, color, odor and overall acceptability, while sample (D) had the highest score values of texture and taste. Moreover, sample $(F)$ had the lowest score of all tested attributes.

From the above results, it could be noticed that nitrite-reduced meat curing mixtures which included sodium nitrite $80 \mathrm{mg} / \mathrm{kg}$, chitosan $10 \mathrm{~g} / \mathrm{kg}$ and rosemary $2000 \mathrm{mg} / \mathrm{kg}$ (sample B), sodium nitrite $80 \mathrm{mg} / \mathrm{kg}$, betanin 3.6 $\mathrm{mg} / \mathrm{kg}$, chitosan $10 \mathrm{~g} / \mathrm{kg}$ and rosemary $2000 \mathrm{mg} / \mathrm{kg}$ (sample D) and sodium nitrite $40 \mathrm{mg} / \mathrm{kg}$, betanin $14.4 \mathrm{mg} / \mathrm{kg}$, chitosan $10 \mathrm{~g} / \mathrm{kg}$ and rosemary 2000 $\mathrm{mg} / \mathrm{kg}$ (sample E) have the color, oxidative stability overall acceptability and microbial stability which are imparted by nitrite to cured-meat products.

\section{REFERENCES}

Aksu, M. I. and Kaya, M. (2001). Some microbiological, chemical and physical characteristics of pastirma marketed in Erzurum. Tur. J. Vet. Anim. Sci., 25, 319-326.

Aksu, M. I., Kaya, M. and Ockerman, H. W. (2005). Effect of modified atmosphere packaging and temperature on the shelf life of sliced pastirma produced from frozen/thawed meat. J. Muscle Foods, 16, 192-206.

Alley, G., Cours, D. and Demeyer, D. (1992). Effect of nitrate, nitrite and ascorbate on color and color stability of dry fermented sausage prepared using 'Back Slopping'. Meat Sci., 32, 279-287.

Amtliche Sammlung von Untersuchungsverfahren; Bekanntmachungen nach $\S 64$ LFGB (1980). Untersuchung von lebensmitteln bestimmung des gesamtfettgehaltes in fleisch und fleischerzeugnissen, L-06.00-6.

Amtliche Sammlung von Untersuchungsverfahren; Bekanntmachungen nach $\S 64$ LFGB (1990). Untersuchung von lebensmitteln bestimmung des nitrit- und nitratgehaltes in wurstwaren nach enzymatischer reduktion, L-08.00-14.

AOAC (1990). Official Methods of Analysis, $15^{\text {th }}$ Ed., Association of Official Analytical Chemists, Helrch, K. (Ed.). Arlington, VA, USA.

Bakr, A. A. and Mahmoud, W. H. (1995). Overall quality, oxidative and microbial stabilities of nitrite-reduced sausages. Zagazig J. Agric. Res., 22, 1023-1031. 
Bloukas, J. G., Arvanitoyannis, I. S. and Siopi, A. A. (1999). Effect of natural colorants and nitrites on color attributes of frankfurters. Meat Sci., 52, 257-265.

Bover-Cid, S., Izquierdo-Pulido, M. and Vidal-Carou, M. C. (1999). Effect of proteolytic starter cultures of Staphylococcus spp. on biogenic amine formation during the ripening of dry fermented sausages. Int. J. Food Microbiol., 46, 95-104.

Bozkurt, H. (2006). Utilization of natural antioxidants: Green tea extract and Thymbra spicata oil in Turkish dry-fermented sausage. Meat Sci., 73, 442-450.

Bozkurt, H. and Erkmen, O. (2007). Effects of some commercial additives on the quality of sucuk (Turkish dry-fermented sausage). Food Chem., 101, 1465-1473.

Byun, M. W., Lee, J. W., Jo, C. and Yook, H. S. (2001). Quality properties of sausage made with gamma-irradiated natural prok and lamb casing. Meat Sci., 59, 223-228.

Chouliara, I., Samelis, J., Kakouri, A., Badeka, A., Savvaidis, I. N., Riganakos, K. and Kontominas, M. G. (2006). Effect of irradiation of frozen meat/fat trimmings on microbiological and physicochemical quality attributes of dry fermented sausages. Meat Sci., 74, 303-311.

Darmadji, P. and Izumimoto, M. (1994). Effect of chitosan in meat preservation. Meat Sci., 38, 243-254.

Decker, A. E., Chan, W. K. M., Livisay, S. A., Butterfield, D. A. and Faustman, C. (1995). Interaction between carnosine and the different redox states of myoglobin. J. Food Sci., 60, 1201-1204.

Egyptian Standard (1991). Meat and meat products (pastirma). Standard Bulletin No 1042. Egyptian organization for standardization and quality control, Ministry of Industry, Cairo, Egypt.

Georgantelis, D., Blekas, G., Katikou, P., Ambrosiadis, I. and Fletouris, D. J. (2007). Effect of rosemary extract, chitosan and a-tocopherol on lipid oxidation and color stability during frozen storage of beef burgers. Meat Sci., 75, 256-264.

Hernández-Jover, T., Izquierdo-Pulido, M., Veciana-Nogués, M. T., MarinéFont, A. and Vidal-Carou, M. C. (1997). Effect of starter cultures on biogenic amine formation during fermented sausage production. J. Food Prot., 60, 825-830.

Honikel, K.-O. (2008). The use and control of nitrate and nitrite for the processing of meat products. Meat Sci., 78, 68-76.

Kayaardi, S. and Gök, V. (2003). Effect of replacing beef fat with olive oil on quality characteristics of Turkish soudjouk (sucuk). Meat Sci., 66, 249257.

Komprda, T., Neznalovà, J., Standara, S. and Bover-Cid, S. (2001). Effect of starter culture and storage temperature on the content of biogenic amines in dry fermented sausage poličan. Meat Sci., 59, 267-276.

Marco, A., Navarro, J. L. and Flores, M. (2006). The influence of nitrite and nitrate on microbial, chemical and sensory parameters of slow dry fermented sausage. Meat Sci., 73, 660-673. 
Mitsumoto, M., O'Grady, M. N., Kerry, J. P. and Buckley, D. J. (2005). Addition of tea catechins and vitamin $\mathrm{C}$ on sensory evaluation, color and lipid stability during chilled storage in cooked or raw beef and chicken patties. Meat Sci., 69, 773-779.

Pérez-Alvarez, J. A., Sayas-Barberá, E., Fernández-López, J. and ArandaCatalá, V. (1999). Physicochemical characteristics of Spanish-type drycured sausage. Food Res. Int., 32, 599-607.

Samelis, J., Metaxopoulos, J., Vlassi, M. and Pappa, A. (1998). Stability and safety of traditional Greek salami-a microbiological ecology study. Int. J. Food Microbiol., 44, 69-82.

Schweinsberg, F. and Bürkle, V. (1985). Nitrite: A co-carcinogen? J. Cancer Res. Clin. Oncol., 109, 200-202.

Shahidi, F. and Pegg, R. B. (1992). Nitrite-free meat curing systems: Update and review. Food chem., 43, 185-191.

Shahidi, F., Rubin, L. J. and Wood, D. F. (1987). Control of lipid oxidation in cooked ground pork with antioxidants and dinitrosyl ferrohemochrome. J. Food Sci., 52, 564-567.

Shehata, H. A. (1989). Studies on nitrate and nitrite in meat products. Ph.D. thesis, Food Science and Technology Department, Faculty of Agriculture, Suez Canal University, Ismailia, Egypt.

Strotmann, C., von Mueffling, T., Klein, G. and Nowak, B. (2008). Effect of different concentrations of carbon dioxide and oxygen on the growth of pathogenic Yersinia enterocolitica 4/0:3 in ground pork packaged under modified atmospheres. J. Food Prot., 71, 845-849.

Stuempel, S. (1997). Eigenschaften von mit karmin, betanin und beta-corotin gefaerbter bruehwurst. Fleischwirtschaft, 77, 435-439.

Tyrpenou, A. E., Gouta, E. H., Tsigouri, A. D. and Vlasiotis, C. N. (2000). Nitrite and nitrate residual in Greek pastirma. Bulletin Hell. Vet. Med. Soc., 51, 302-307.

Zarringhalami, S., Sahari, M. A. and Hamidi-Esfehani, Z. (2009). Partial replacement of nitrite by annatto as a color additive in sausage. Meat Sci., 81, 281-284.

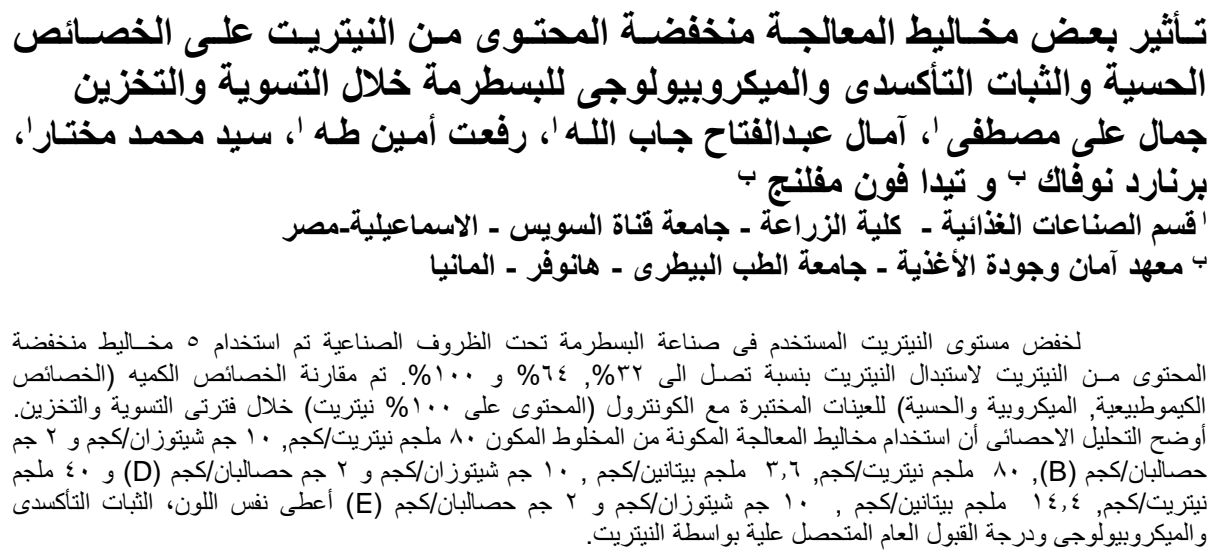


J. Agric. Sci. Mansoura Univ., 34 (7), July, 2009 
Table (1). Effect of different curing mixtures on $L, a$ and $b$ values of pastirma during ripening and storage.

\begin{tabular}{|c|c|c|c|c|c|c|c|c|c|}
\hline \multirow{2}{*}{\begin{tabular}{|r} 
Days \\
Treatments \\
\end{tabular}} & \multirow{2}{*}{ Parameter } & \multicolumn{4}{|c|}{ Ripening time (days) } & \multicolumn{4}{|c|}{ Storage time (days) } \\
\hline & & 0 & 1 & 8 & 17 & 0 & 30 & 60 & 90 \\
\hline \multirow{3}{*}{ A } & $L$ & $41.09 \pm 0.33 a$ & $40.98 \pm 0.35 a$ & $40.57 \pm 0.21 \mathrm{a}$ & $40.32 \pm 0.41 a$ & $40.32 \pm 0.41 \mathrm{a}$ & $39.29 \pm 0.35 a$ & $37.78 \pm 0.28 a$ & $36.32 \pm 0.24 a$ \\
\hline & a & $18.04 \pm($ & $1821 \pm 0.28 a$ & $20.92 \pm 0.24 a$ & $35.29 \pm 0.26 a$ & $35.29 \pm 0.26 a$ & $33.89 \pm 0.91 a$ & $31.62 \pm 0.40 \mathrm{a}$ & $30.65 \pm 0.50 a$ \\
\hline & $\boldsymbol{b}$ & $17.08 \pm 0.29 a$ & $17.02 \pm 0.29 a$ & $16.95 \pm 0.20 \mathrm{a}$ & $16.74 \pm 0.38 a$ & $16.74 \pm 0.38 a$ & $15.34 \pm 0.28 a$ & $14.77 \pm 0.14 \mathrm{a}$ & $13.99 \pm 0.18 a$ \\
\hline \multirow{3}{*}{ B } & $L$ & $41.02 \pm 0.26 a$ & $41.01 \pm 0.26 a$ & $40.61 \pm 0.18 a$ & $40.36 \pm 0.23 a$ & $40.36 \pm 0.23 a$ & $39.10 \pm 0.27 a$ & $37.51 \pm 0.28 a$ & $36.11 \pm 0.21 a$ \\
\hline & $a$ & $17.84 \pm 0.45 a$ & $17.92 \pm 0.42 a$ & $20.71 \pm 0.10 a$ & $35.03 \pm 0.24 a$ & $35.03 \pm 0.24 a$ & $33.26 \pm 0.48 a$ & $31.07 \pm 0.57 a b$ & $30.44 \pm 0.79 a b$ \\
\hline & $\boldsymbol{b}$ & $16.74 \pm 0.35 a$ & $16.70 \pm 0.28 a$ & $16.57 \pm 0.24 a$ & $16.02 \pm 0.26 a$ & $16.02 \pm 0.26 a$ & $15.29 \pm 0.35 a$ & $14.69 \pm$ & $0.14 a$ \\
\hline \multirow{3}{*}{ C } & $L$ & $40.83 \pm 0$. & $40.85 \pm 0.14 \mathrm{a}$ & $40.60 \pm 0.16 a$ & $40.00 \pm 0.35 a b$ & $40.00 \pm 0$ & $38.92 \pm C$ & 36. & $28 \mathrm{a}$ \\
\hline & $a$ & $17.88 \pm 0$ & $17.99 \pm 0.35 a$ & $20.11 \pm 0.13 b$ & $0.47 b$ & $32.23 \pm 0.47 b$ & $31.53 \pm 0.5$ & $.71 b$ & \\
\hline & $b$ & $16.45 \pm 0.42 \mathrm{a}$ & & $16.21 \pm 0.30 a$ & & $15.86 \pm$ & & & \\
\hline \multirow{3}{*}{ D } & $L$ & $40.26 \pm 0.07 \mathrm{~b}$ & $40.12 \pm 0.14 b$ & $39.86 \pm 0.20 \mathrm{~b}$ & $39.19 \pm 0.27 b$ & $39.19 \pm 0.27 b$ & $38.06 \pm 0.35 b$ & $35.80 \pm 0.14 b$ & $0.21 \mathrm{~b}$ \\
\hline & $a$ & $24.85 \pm 0.28 b$ & $24.91 \pm 0.21 b$ & $25.99 \pm 0.35 \mathrm{~cd}$ & $33.79 \pm 0.69 \mathrm{ab}$ & $33.79 \pm 0.69 \mathrm{ab}$ & $32.06 \pm 0.99 a b$ & $30.85 \pm 0.71 \mathrm{ab}$ & $29.68 \pm 0.57 \mathrm{ab}$ \\
\hline & $b$ & & $18.31 \pm 0.42 b$ & $18.02 \pm 0.11 \mathrm{~b}$ & $17.98 \pm 0.28 b$ & $17.98 \pm 0.28 b$ & $16.36 \pm 0.07 b$ & $15.21 \pm 0.07 b$ & $14.53 \pm 0.12 b$ \\
\hline \multirow{3}{*}{ E } & $L$ & $39.76 \pm 0.28 b$ & $39.70 \pm 0.21 b$ & $39.41 \pm 0.10 \mathrm{~b}$ & $38.41 \pm 0.23 c$ & $38.41 \pm 0.23 c$ & $37.71 \pm 0.18 b$ & $35.11 \pm 0.27 \mathrm{c}$ & $34.72 \pm 0.07 \mathrm{c}$ \\
\hline & $a$ & $26.10 \pm 0.21 \mathrm{c}$ & $26.14 \pm 0.28 c$ & $26.41 \pm 0.20 c$ & $36.01 \pm 0.85 a$ & $36.01 \pm 0.85 a$ & $34.33 \pm 0.57 a$ & $32.19 \pm 0.55 a$ & $30.97 \pm 0.31 a$ \\
\hline & $b$ & $19.14 \pm 0.37 \mathrm{bc}$ & $19.11 \pm 0.35 b c$ & $19.01 \pm 0.20 c$ & $18.58 \pm 0.07 \mathrm{c}$ & $18.58 \pm 0.07 \mathrm{c}$ & $16.92 \pm 0.07 \mathrm{c}$ & $15.81 \pm 0.21 \mathrm{c}$ & $14.96 \pm 0.14 \mathrm{c}$ \\
\hline \multirow{2}{*}{ I } & $L$ & $39.02 \pm 0.14 \mathrm{c}$ & $39.00 \pm 0.14 \mathrm{c}$ & $38.62 \pm 0.16 c$ & $37.53 \pm 0.35 d$ & $37.53 \pm 0.35 d$ & $37.05 \pm 0.10 \mathrm{c}$ & $34.89 \pm 0.09 c$ & $33.61 \pm 0.16 d$ \\
\hline & $a$ & $29.89 \pm 0.98 d$ & $29.71 \pm 0.92 d$ & $25.30 \pm 0.06 \mathrm{~d}$ & $22.30 \pm 0.64 \mathrm{c}$ & $22.30 \pm 0.64 c$ & $20.86 \pm 0.28 c$ & $19.61 \pm 0.14 \mathrm{c}$ & $18.54 \pm 0.48 c$ \\
\hline
\end{tabular}


\begin{tabular}{l|l|l|l|l|l|l|l|l|}
$\boldsymbol{b}$ & $19.97 \pm 0.18 \mathrm{c}$ & $19.95 \pm 0.14 \mathrm{c}$ & $19.61 \pm 0.27 \mathrm{c}$ & $18.99 \pm 0.12 \mathrm{~d}$ & $18.99 \pm 0.12 \mathrm{~d}$ & $17.31 \pm 0.14 \mathrm{~d}$ & $16.36 \pm 0.15 \mathrm{~d}$ & $15.25 \pm 0.07 \mathrm{~d}$ \\
\hline
\end{tabular} Values with different letters in each column are significantly different $(\mathrm{p}<0.05)$ from one to another. (A: control sodium nitrite $125 \mathrm{mg} / \mathrm{kg}, \mathrm{B}$ : sodium nitrite $80 \mathrm{mg} / \mathrm{kg}+$ chitosan $10 \mathrm{~g} / \mathrm{kg}+$ rosemary $2 \mathrm{~g} / \mathrm{kg}$, C: sodium nitrite $40 \mathrm{mg} / \mathrm{kg}+$ chitosan $10 \mathrm{~g} / \mathrm{kg}+$ rosemary $2 \mathrm{~g} / \mathrm{kg}$, D: sodium nitrite $80 \mathrm{mg} / \mathrm{kg}+$ betanin $3.6 \mathrm{mg} / \mathrm{kg}+$ chitosan $10 \mathrm{~g} / \mathrm{kg}+$ rosemary $2 \mathrm{~g} / \mathrm{kg}$, E: sodium nitrite $40 \mathrm{mg} / \mathrm{kg}+$ betanin $14.4 \mathrm{mg} / \mathrm{kg}+\mathrm{chitosan} 10 \mathrm{~g} / \mathrm{kg}+$ $80 \mathrm{mg} / \mathrm{kg}+$ betanin $3.6 \mathrm{mg} / \mathrm{kg}+\mathrm{chitosan} 10 \mathrm{~g} / \mathrm{kg}+$ rosemary $2 \mathrm{~g} / \mathrm{kg}$, E: sodic
rosemary $2 \mathrm{~g} / \mathrm{kg}$, F: betanin $21.6 \mathrm{mg} / \mathrm{kg}$ + chitosan $10 \mathrm{~g} / \mathrm{kg}$ + rosemary $2 \mathrm{~g} / \mathrm{kg}$ ). 